\title{
Advanced Therapy for Inflammatory Bowel Disease: A Guide for the Primary Care Physician
}

\author{
Arul Thomas, MD, and Nilesh Lodhia, MD
}

Primary care physicians care for patients with inflammatory bowel disease (IBD) who are receiving advanced therapies that include immunomodulator drugs (eg, azathioprine and methotrexate) and biologic therapy. These agents have significantly improved remission rates and the quality of life for patients suffering from IBD. However, patients taking these drugs need special care and counseling with regard to adverse effects, infection risk, cancer risk, and pregnancy. Newer treatment paradigms incorporate earlier use of biologic therapy, often in combination with immunomodulator drugs, to alter the natural course of the disease. Comprehensive care for these patients, including health maintenance, requires collaboration between primary care physicians and gastroenterologists. Despite their high cost, advanced therapies are likely to be cost-effective. This article discusses general concepts about azathioprine, 6-mercaptopurine, methotrexate, and common biologic drugs used in IBD. (J Am Board Fam Med 2014;27:411-420.)

Keywords: Inflammatory Bowel Diseases, Immunomodulatory Therapy, Biologic Therapy

Primary care physicians will care for patients with inflammatory bowel disease (IBD) who are receiving advanced therapies that include immunomodulator and biologic therapy (Table 1). These agents significantly improve remission rates and quality of life for patients suffering from IBD. However, patients taking these drugs need special care and counseling with regard to adverse effects, infection risk, cancer risk, and pregnancy. The purpose of this article is to provide background on these medications to the primary care provider. The need for systemic steroids should prompt gastroenterologists to consider immunomodulator or biologic therapy for optimal disease control. While systemic steroids classically are used to treat acute exacerba-

This article was externally peer reviewed.

Submitted 1 August 2013; revised 27 October 2013; accepted 4 November 2013.

From the Division of Gastroenterology and Hepatology, Medical University of South Carolina, Charleston.

Funding: none.

Conflict of interest: none declared.

Corresponding author: Nilesh Lodhia, MD, Division of Gastroenterology and Hepatology, Medical University of South Carolina, 25 Courtenay Drive, ART 7100A, MSC 290, Charleston, SC 29425 (E-mail: lodhia@musc.edu). tions of IBD, they are ineffective in maintaining remission. ${ }^{1}$ Furthermore, the side effect profile of corticosteroids prohibits their long-term use.

The traditional treatment algorithm for IBD follows a step-wise treatment approach, with intensified treatments used in sequence if remission is not achieved with an earlier treatment (Figure 1). Recent data, however, suggests that the early initiation of biologic therapy can modify disease progression. The efficacy of biologics has shifted the treatment paradigm, allowing gastroenterologists to incorporate more aggressive treatments early in the course of disease to alter the natural history of IBD. Immunomodulator drugs often are combined with biologics in patients with aggressive disease; a recent landmark study has illustrated a synergistic effect of these agents, ${ }^{2-5}$ which can be used indefinitely to maintain remission.

\section{Immunomodulator Therapy}

Thiopurines and methotrexate are commonly used as immunomodulator therapies. Their mechanisms of action, metabolism, efficacy, and side effects are described here. 


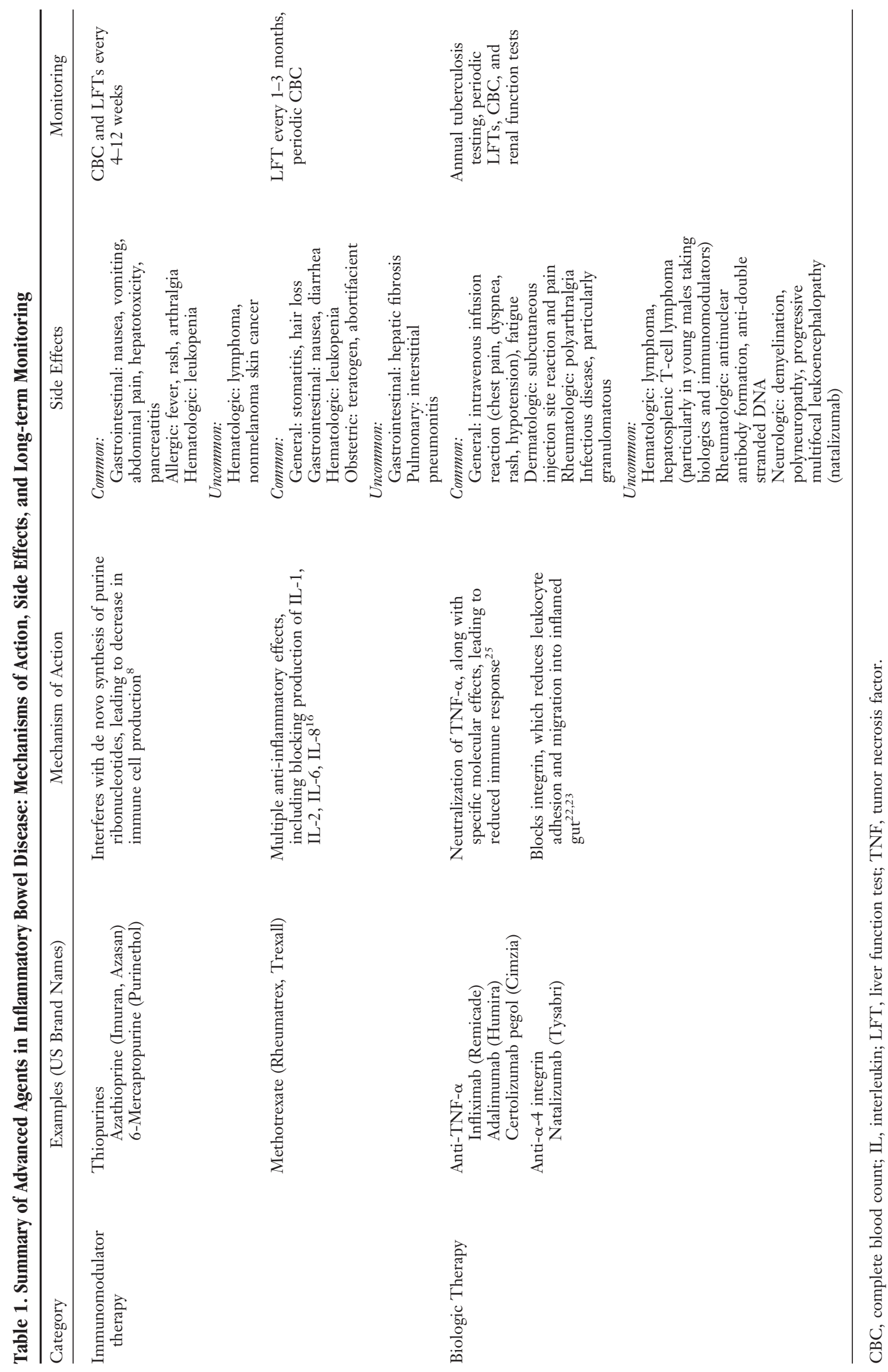

412 JABFM May-June 2014 Vol. 27 No. 3 
Figure 1. A simplified approach to "step-wise" treatment of inflammatory bowel disease. 5-ASA, 5aminosalicylic acid.

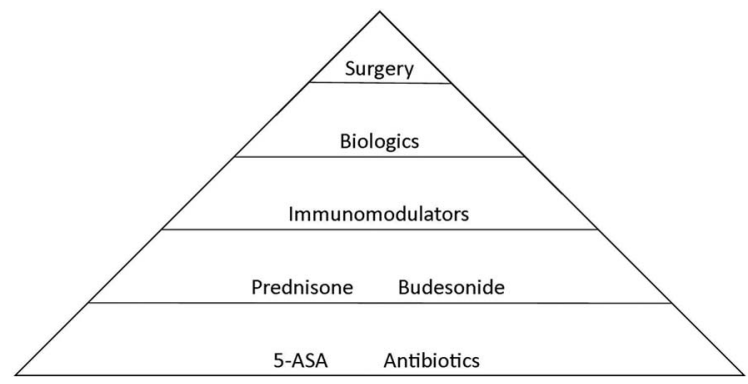

\section{Thiopurines}

\section{Action and Metabolism}

The thiopurine analogues azathioprine and 6-mercaptopurine (6-MP) gained widespread acceptance as established treatments for IBD in the early 1980s, although azathioprine's efficacy in healing fistulas had been reported over a decade prior. These medications work through multiple mechanisms to control the dysregulated immune response in IBD. The active thiopurine metabolite 6-thioguanine nucleotide is a purine antagonist and therefore interferes with DNA and RNA synthesis. The reduction in DNA and RNA synthesis inhibits the proliferation of $\mathrm{T}$ and $\mathrm{B}$ lymphocytes.
The variation of thiopurine metabolism among patients guides therapy for both azathioprine and 6-MP. Azathioprine is converted to $6-\mathrm{MP}$ by a nonenzymatic reaction occurring within erythrocytes. Primary understanding of this metabolism begins with the thiopurine S-methyltransferase (TPMT) enzyme (Figure 2). There is significant genetic variation in TPMT enzymatic activity, and determining enzyme activity before initiation can help guide dosing. TPMT testing, however, does not preclude the need for monitoring for hepatotoxicity or leukopenia. While enzyme testing is expensive, it has been shown to reduce long-term costs from inappropriate dosing. ${ }^{6,7}$

\section{Efficacy}

Azathioprine and 6-MP promote clinical remission and steroid sparing in patients. ${ }^{8} \mathrm{~A}$ recent Cochrane Database Systemic Review showed an odds ratio of 2.43 (95\% confidence interval [CI], 1.62-3.64) for response in patients with Crohn's disease who were treated with azathioprine or 6-MP compared with placebo. ${ }^{9}$ The steroid sparing effect was significant, with an odds ratio of 3.69 (95\% CI, 2.12-6.42). Earlier data estimated that one half to two thirds of patients will respond to thiopurine treatment. ${ }^{8}$ Thiopurines have a delayed onset of action, requir-

Figure 2. A simplified approach to azathioprine (AZA) metabolism. Thiopurine methyltransferase (TPMT) breaks down 6-mercaptopurine (6-MP) into the hepatotoxic metabolite 6-methylmercaptopurine (6-MMP). Besides TPMT metabolism, there are 2 other major pathways from 6-MP that should be considered. One is driven by the hypoxanthine phosphoribosyl transferase (HPRT) enzyme, leading to 6-thioguanine nucleotide (6-TGN), the metabolite responsible for both the therapeutic benefit in inflammatory bowel disease and myelosuppression. The other pathway is driven by xanthine oxidase (XO), leading to production of 6-thiouric acid (6-TU), an inactive metabolite. 6-TIMP, 6-thioinosine monophosphate.

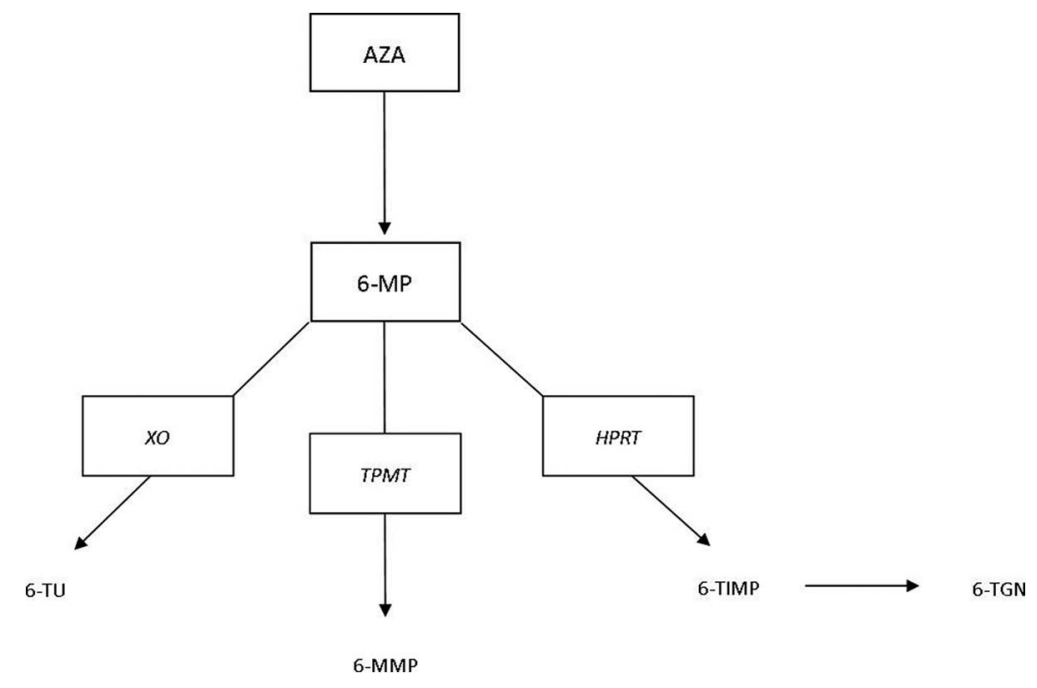


ing at least 3 to 4 months for a clinical effect to occur. $^{8}$

\section{Side Effects}

The side effect profiles of azathioprine and 6-MP are significant, and $9.3 \%$ of patients develop adverse effects serious enough to stop therapy (Table 1). ${ }^{8}$ Allergic reactions include fever, rash, arthralgias, and pancreatitis; these occur independent of dose and resolve with discontinuation of the drug. Acute pancreatitis can be seen in 3\% to $7 \%$ of patients, typically during the first month of treatment. Chronic pancreatitis attributable to azathioprine or 6-MP has not been reported. ${ }^{10,11}$ Switching between azathioprine and 6-MP may help obviate side effects. However, patients who develop acute pancreatitis while taking either agent should be considered intolerant to both medications.

Myelosuppression is an important and potentially lethal complication of thiopurine therapy, and the white cell line is most commonly affected. Although typically associated with low TPMT enzyme activity, myelosuppression can also occur with normal enzymatic activity. Hepatotoxicity can be seen in up to $2 \%$ of patients and is typically caused by increased synthesis of 6-methylmercaptopurine (Figure 2). ${ }^{12}$ Both myelosuppression and hepatotoxicity are dose-dependent responses, and management consists of dose reduction and possibly drug cessation.

For many patients and physicians, the most alarming adverse effect associated with thiopurine therapy is malignancy; the strongest associations have been linked with lymphoma and nonmelanoma skin cancer. A recent analysis of almost 20,000 French patients suggested that the risk of lymphoma in patients with IBD who were receiving thiopurines increased from 0.26 to 0.9 per 1000 patient-years, with a multivariate hazard ratio of 5.28 (95\% CI, 2.01-13.9). ${ }^{13}$ Furthermore, there have been 36 case reports of hepatosplenic T-cell lymphoma associated with thiopurine use, which most commonly occurs in young men and is usually fatal; 20 of these cases were associated with the concomitant use of biologic therapy, and 16 involved thiopurine use alone. ${ }^{14} \mathrm{~A}$ study of patients taking thiopurines for $>1$ year showed a relative risk of 4.27 (95\% CI, 3.08-5.92) for the development of nonmelanoma skin cancer. ${ }^{15}$ The risk was also high in patients taking anti-tumor necrosis factor (TNF) biologics and further increased in those taking dual therapy with thiopurines and anti-TNF biologics. ${ }^{15}$ Patients should ensure regular use of sunscreen during sun exposure and have annual skin exams by their primary care provider or dermatologist.

Except for special circumstances, thiopurines should not be used with xanthine oxidase inhibitors such as allopurinol or febuxostat. Furthermore, caution must be exercised with concomitant use of warfarin (may decrease the efficacy of warfarin) or angiotensin-converting enzyme inhibitors (may increase the risk of myelosuppression). However, both warfarin and angiotensin-converting enzyme inhibitors can be continued with thiopurines as long as blood work is monitored.

\section{Methotrexate}

Action

Methotrexate was pioneered for the treatment of rheumatoid arthritis in the 1950s. It should be considered an alternative to thiopurines. A clinical decision to use methotrexate is influenced by its side effect profile, namely teratogenicity, and its intramuscular or subcutaneous forms of administration. Methotrexate has numerous anti-inflammatory effects, including blocking production of interleukin (IL)-1, IL-2, IL-6, and IL-8. ${ }^{16}$

\section{Efficacy}

Randomized controlled trials have shown the efficacy of methotrexate in the induction and maintenance of remission in Crohn's disease. ${ }^{17-19}$ Based solely on existing data, methotrexate cannot be considered a major treatment for ulcerative colitis. For active ulcerative colitis, a single randomized controlled trial including 67 patients showed similar remission rates after 4 months between the oral methotrexate group and the placebo group. ${ }^{20}$ However, this study was limited in size and its use of oral methotrexate. In clinical practice, methotrexate is frequently successful in treating ulcerative colitis. A large randomized controlled trial is currently ongoing to determine the efficacy of highdose subcutaneous methotrexate in patients with ulcerative colitis. A clinical response can be expected within 8 weeks of starting therapy. ${ }^{17}$

\section{Side Effects}

Although usually well-tolerated, the side effect profile of methotrexate includes nausea, stomatitis, diarrhea, hair loss, leukopenia, interstitial pneumoni- 
tis, and hepatic fibrosis (Table 1). Nausea is the most common side effect and usually improves with time. It is frequently managed supportively with ondansetron. Furthermore, daily folic acid can reduce nausea as well as stomatitis. Although the risk of hepatic fibrosis is low in patients with IBD, cirrhosis is the most worrisome adverse effect of methotrexate. The risk of cirrhosis is directly related both to the cumulative exposure to methotrexate as well as the presence of other risk factors for liver disease. ${ }^{21}$ Therefore, patients with a history of excessive alcohol use and nonalcoholic fatty liver risk factors (eg, diabetes, obesity, hyperlipidemia) should avoid methotrexate. ${ }^{21}$ Elevated aminotransferase levels do not always correlate with the presence of hepatic fibrosis, and a liver biopsy should be considered if there is reasonable clinical suspicion for hepatic fibrosis, particularly if the cumulative dose has exceeded $1.5 \mathrm{~g} .{ }^{21}$ Methotrexate has high abortifacient and teratogenic effects, and patients should be counseled appropriately.

In general, potentially hepatotoxic and myelosuppressive medications should be avoided with methotrexate. Furthermore, the concurrent use of nonsteroidal anti-inflammatory drugs can increase methotrexate concentrations, thus increasing the risk of methotrexate toxicity.

\section{Biologic Therapy Actions and Characteristics}

Infliximab, adalimumab, and certolizumab pegol are 3 biologic agents that target TNF activity, decreasing mucosal inflammation through multiple mechanisms. Infliximab is a chimeric immunoglobulin (Ig) G antibody that binds to TNF, and in the late 1990s it was the first biologic approved for use in IBD. It is administered intravenously. Adalimumab is a humanized IgG antibody that binds to TNF and is administered via subcutaneous injections. Certolizumab pegol is a pegylated fragment of the IgG antibody and also is given as a subcutaneous injection.

Natalizumab blocks leukocyte adhesion to and migration into areas of inflammation. As a humanized monoclonal antibody, it targets the protein $\alpha-4$ integrin. Data have shown efficacy for moderate to severe Crohn's disease, but concerns over safety have prevented routine use. ${ }^{22,23}$ In patients with multiple sclerosis, reports of progressive multifocal leukoencephalopathy associated with the
John Cunningham (JC) virus have been described. ${ }^{24}$ This risk, along with hepatotoxicity, has reserved use of this biologic for only specific cases. Antibody testing for JC virus before therapy is recommended. The remainder of the discussion on biologic therapy is devoted to antiTNF agents.

\section{Efficacy}

Sixty percent of patients will clinically respond to biologic treatment within 2 to 6 weeks of initiation. ${ }^{25}$ Multiple trials have shown that induction dosing with regular maintenance dosing, compared with intermittent dosing based on symptoms, ensures the highest efficacy and prevents loss of response. Patients with shorter disease duration benefit the most from biologics. ${ }^{25}$ Nonetheless, response declines in $30 \%$ to $50 \%$ of initial responders while on maintenance therapy, within 1 to 3 years. ${ }^{25}$ Loss of response can be attributed to the formation of antibodies, altered pharmacokinetics, or changes in the dominant mechanism of inflammation. ${ }^{25}$ Antibody and metabolite testing of anti-TNF agents will better define and standardize loss of response. When patients decompensate clinically without evidence of active inflammation on endoscopy, other processes such as a stricture, enteric infection (eg, Clostridium difficile), and concomitant irritable bowel syndrome should be considered. Biologic treatment also has been found to be efficacious in the long-term treatment of fistulas associated with Crohn's disease. ${ }^{26-28}$

\section{Side Effects}

Reactions at the sites of subcutaneous injection (adalimumab and certolizumab) and intravenous infusion (infliximab) can occur during biologic therapy. Patients who have developed anti-infliximab antibodies are most prone to infusion reactions and can present with a syndrome of chest pain, dyspnea, rash, and hypotension. ${ }^{26,29} \mathrm{~A}$ delayed hypersensitivity reaction, occurring within a few days to 2 weeks after infusion, can also occur. Symptoms include severe polyarthralgia, myalgia, facial edema, urticaria, and rash. ${ }^{30}$ General management includes supportive care and a short course of oral steroids. Infections are a dreaded complication of biologic therapy, and the use of concomitant immunosuppressants can increase infection risk. There is an overall $2 \%$ to $4 \%$ risk of serious infection in the major trials of the biologics 
discussed. ${ }^{30-32}$ Fungal, atypical, and mycobacterial (eg, reactivation of tuberculosis) infections should be considered in the workup of these patients. A chest radiograph along with hepatitis B and tuberculosis testing are mandatory before beginning treatment. Data on whether biologic therapy poses an increased risk for lymphoma is conflicting, but the preponderance of the data suggests that the increased risk for lymphoma from IBD therapy is principally attributable to thiopurines. ${ }^{33,34}$ The formation of antinuclear antibodies and anti-double stranded DNA can also occur with use of antiTNF biologic therapy over the long-term. ${ }^{4} \mathrm{Al}$ though drug-induced lupus is an uncommon side effect, the mere presence of antibodies is not pathogenic. Central and peripheral demyelination and polyneuropathy are uncommon neurologic side effects of anti-TNF biologic therapy. ${ }^{35}$

Biologic therapies have few absolute drug-drug interactions. However, the risks and benefits of concomitant immunosuppressant use should be carefully considered.

\section{Long-Term Considerations with Advanced Therapies \\ Long-term Follow-up}

Hematologic and liver function need to be closely monitored during initiation and escalation of thiopurines but less frequently monitored after a stable dose has been reached. Although published guidelines and clinical practices vary considerably, a reasonable approach is to perform a complete blood count and liver function tests every 2 weeks for 6 weeks and then every 4 to 12 weeks after that period. ${ }^{36-39}$ Testing for 6-thioguanine nucleotide and 6-methylmercaptopurine metabolites can help guide dosage adjustments over the long-term. Methotrexate monitoring should involve liver function tests every 1 to 3 months and periodic complete blood count testing.

Biologic therapy does not require absolute monitoring. If a patient's clinical picture suggests worsening disease, analysis of trough level and antibodies (for infliximab and adalimumab) can help determine whether the drug is still effective. ${ }^{25}$ These laboratory tests are not available commercially for certolizumab pegol. Patients should have yearly tuberculous skin testing or serum interferon-gamma release assay testing.

\section{Pregnancy and Family Planning}

Many patients with IBD are affected during their peak reproductive years. Thiopurines are classified as Federal Drug Administration (FDA) pregnancy category $\mathrm{D}$, but this classification is based on teratogenicity in animals. The preponderance of human data suggests that thiopurines are not associated with a significant increase in the rates of prematurity, spontaneous abortion, congenital defects, neonatal infections, or neoplasia. ${ }^{40-42}$ Given the known correlation of active IBD with adverse neonatal outcomes, the risks of discontinuing thiopurines in a patient with IBD before (or during) pregnancy generally outweigh the benefits. ${ }^{43} \mathrm{Al}$ though thiopurine can be transferred through the placenta, the transfer of the drug via lactation is minimal, particularly after the first 4 hours of drug intake. Therefore, breastfeeding is generally considered safe if performed 4 to 6 hours after ingestion of azathioprine or 6-MP. ${ }^{44-48}$

Methotrexate is a well-established teratogen and cannot be used in women considering pregnancy. ${ }^{49}$ It is FDA pregnancy category X. Methotrexate embryopathy occurs during the critical period of organogenesis (6-8 weeks after conception) but can occur during the second and third trimester, too. Intrauterine growth retardation, decreased ossification of the calvarium, low-set ears, micrognathia, limb deformities, and mental retardation occur. Because of its penetration into tissue, women of childbearing age who are taking methotrexate should use 2 forms of birth control and wait 6 months after stopping the drug before trying to conceive. Breastfeeding while taking methotrexate is also contraindicated.

Infliximab, adalimumab, and certolizumab pegol are FDA pregnancy category B drugs and are generally considered safe for use during pregnancy. ${ }^{41}$ Despite their overall safety during pregnancy, infliximab and adalimumab cross the placenta in the second and third trimester and are detectable in infants for up to 7 months after birth. ${ }^{50,51}$ Although low concentrations of infliximab and adalimumab are generally considered safe for infants, caution should be exercised when considering the rotavirus vaccine in these infants. ${ }^{50,52,53}$ For this reason, some experts recommend that infliximab and adalimumab be stopped at gestational week $30 .{ }^{44}$ However, this should be an individualized decision with input from the patient, primary care physician, gastroenterologist, and obstetrician. Certolizumab 
Table 2. Examples of Doses and Cost per Dose of Common Advanced Therapies for Inflammatory Bowel Disease

\begin{tabular}{lrr}
\hline Examples by Category (US Brand Names) & Dose $^{*}$ & ${\text { Cost per Dose }(\$)^{\dagger}}^{\text {Immunomodulator therapy }}$ \\
Thiopurines & & \\
$\quad$ Azathioprine (Imuran, Azasan) & $100 \mathrm{mg}$ daily (generic, supply of 50 tablets) & 262.16 \\
$\quad$ Methotrexate (Rheumatrex, Trexall) & $25 \mathrm{mg} / \mathrm{mL}$ per week (generic, supply 2 mL solution) & 8.59 \\
Biologic therapy & & 974.27 \\
Anti-tumor necrosis factor- $\alpha$ & $100 \mathrm{mg}$ intravenous every 8 weeks (brand, one dose) & 2809.30 \\
$\quad$ Infliximab (Remicade) & $40 \mathrm{mg}$ subcutaneous every 2 weeks (brand, 2 doses) \\
$\quad$ Adalimumab (Humira) & $400 \mathrm{mg}$ subcutaneous once monthly (brand, one dose) & 2664.00 \\
$\quad$ Certolizumab pegol (Cimzia) & & 5296.50 \\
Anti- $\alpha-4$ integrin & $300 \mathrm{mg}$ intravenous once monthly (brand, one dose) \\
$\quad$ Natalizumab (Tysabri)
\end{tabular}

${ }^{*}$ Doses reflect only common doses and should not be used to determine starting doses.

${ }^{\dagger}$ Prices obtained from Medi-Span database (www.medispan.com) in October 2013.

pegol is a fragment of an antibody and therefore can be used throughout pregnancy without significant placental transfer. ${ }^{54}$ Certolizumab, as well as infliximab and adalimumab, are considered safe to use while breastfeeding. ${ }^{55}$

Thiopurines and biologic therapies do not affect male fertility, and pregnancy outcomes are not adversely affected if the father is taking these medications. ${ }^{55-58}$ However, men taking sulfasalazine or methotrexate should be warned of reversible oligospermia when trying to conceive. Fertility should be restored in men who stop taking methotrexate or sulfasalazine 3 months before trying to conceive. $^{59,60}$

\section{Health Maintenance Measures}

Primary care physicians and gastroenterologists need to work in tandem to cover the health maintenance needs of patients with IBD. Issues surrounding appropriate vaccinations should be addressed at the time of diagnosis. Live vaccines, including measles-mumps-rubella, varicella, and varicella zoster, are safe to use 4 to 12 weeks before immunosuppression. ${ }^{61}$ Inactivated vaccines can be given during immunosuppression but have a greater serologic response before immunosuppression. ${ }^{62}$ Tetanus/diphtheria, human papillomavirus, pneumococcal, hepatitis A, hepatitis B, meningococcal, and annual influenza vaccines should be considered as appropriate. Female patients with IBD, particularly those taking immunosuppressants, have an increased risk of cervical neoplasia; they are considered high risk and should undergo screening for cervical cancer per the American College of Gynecology guidelines. ${ }^{63,64}$

\section{Approximate Costs}

Both patients and primary care providers should be aware of the relatively high costs of advanced IBD therapies (Table 2). Fortunately, the manufacturers of these drugs have patient-assistance programs, which offer discounted or free therapies to eligible patients. Furthermore, it is important to consider the long-term cost-effectiveness of these expensive medicines, particularly biologic therapies. Achieving and maintaining remission can decrease the need for major surgery and inpatient hospitalizations, thus increasing patients' productivity in the workplace. ${ }^{65}$ As these therapies become more prevalent, further analyses will better delineate their cost-effectiveness.

\section{Conclusion}

Thiopurines, methotrexate, and biologic agents help increase remission rates and quality of life of patients with IBD. Knowledge of these agents will allow primary care physicians to optimize their care of these complex patients. Current treatment paradigms recommend the earlier use of advanced therapies to alter the disease course and minimize complications. Specific side effects, malignancy risk, and safety during pregnancy of these therapies are important issues that concern patients during primary care and gastroenterology visits. The chronic course of IBD enables primary care physicians to collaborate beneficially with gastroenterol- 
ogists. Primary care physicians are more likely to see the adverse effects of advanced therapies and are in the best position to ensure proper health maintenance for these patients. A collaborative relationship can ensure efficacious care over the longterm.

\section{References}

1. Modigliani R, Mary JY, Simon JF, et al. Clinical, biologic, and endoscopic picture of attacks of Crohn's disease. Evolution on prednisolone. Groupe d'Etude Therapeutique des Affections Inflammatoires Digestives. Gastroenterology 1998;98:811-8.

2. Spurio FF, Aratari A, Margagnoni G, Doddato MT, Papi C. Early treatment in Crohn's disease: do we have enough evidence to reverse the therapeutic pyramid? J Gastrointestin Liver Dis 2012;21:67-73.

3. Maser EA, Villela R, Silverberg MS, Greenberg GR. Association of trough serum infliximab to clinical outcome after scheduled maintenance treatment for Crohn's disease. Clin Gastroenterol Hepatol 2006; 4:1248-54.

4. Vermeire S, Noman M, Van Assche G, Baert F, D'Haens G, Rutgeerts P. Effectiveness of concomitant immunosuppressive therapy in suppressing the formation of antibodies to infliximab in Crohn's disease. Gut 2007;56:1226-31.

5. Colombel JF, Sandborn WJ, Reinisch W, et al. Infliximab, azathioprine, or combination therapy for Crohn's disease. N Engl J Med 2010;362:1383-95.

6. Dubinsky MC, Reyes E, Ofman J, Chiou CF, Wade $\mathrm{S}$, Sandborn WJ. A cost-effective analysis of alternative disease management strategies in patients with Crohn's disease treated with azathioprine or 6mercaptopurine. Am J Gastroenterol 2005;100: 2239-47.

7. Priest VL, Begg EJ, Gardiner SJ, et al. Pharmacoeconomic analyses of azathioprine, methotrexate and prospective pharmacogenetic testing for the management of inflammatory bowel disease. Pharmacoeconomics 2006;24:767-81.

8. Pearson DC, May GR, Fick G, Sutherland LR. Azathioprine for maintaining remission of Crohn's disease. Cochran Database Syst Rev 2000;(2): CD000067.

9. Prefontaine E, Macdonald JK, Sutherland LR. Azathioprine or 6-mercaptopurine for induction of remission in Crohn's Disease. Cochrane Database Syst Rev $2013 ;(4): C D 000545$.

10. Pearson DC, May GR, Fick GH, Sutherland LR. Azathioprine and 6-mercaptopurine in Crohn disease. A meta-analysis. Ann Intern Med 1995;123: $132-42$.

11. Su C, Lichtenstein GR. Treatment of inflammatory bowel disease with azathioprine and 6-mercaptopurine. Gastroenterol Clin North Am 2004;33:209-34.

12. Bouhnik Y, Lemann M, Mary JY, et al. Long-term follow- up of patients with Crohn's disease treated with azathioprine or 6-mercaptopurine. Lancet 1996;347: 215-9.

13. Beaugerie L, Brousse N, Bouvier AM, et al. Lymphoproliferative disorders in patients receiving thiopurines for inflammatory bowel disease: a prospective observational cohort study. Lancet 2009;374: 1617-25.

14. Kotylar DS, Osterman MT, Diamond RH, et al. A systematic review of factors that contribute to hepatosplenic $\mathrm{T}$ cell lymphoma. Clin Gastroenterol Hepatol 2011;9:36-41.e31.

15. Peyrin-Biroulet L, Khosrotehrani K, Carrat F, et al. Increased risk for nonmelanoma skin cancers in patients who receive thiopurines for inflammatory bowel disease. Gastroenterology 2011;141:1621-8.

16. Cronstein BN, Naime D, Ostad E. The antiinflammatory mechanism of methotrexate. Increased adenosine release at inflamed sites diminishes leukocyte accumulation in an in vivo model of inflammation. J Clin Invest 1993;92:2675-82.

17. Feagan BG, Rochon J, Fedorak RN, et al. Methotrexate for the treatment of Crohn's disease. The North American Crohn's Study Group Investigators. N Engl J Med 1995;332:292-7.

18. Feagan BG, Fedorak RN, Irvine EJ, et al. A comparison of methotrexate with placebo for the maintenance of remission in Crohn's disease. North American Crohn's Study Group Investigators. N Engl J Med 2000;342:1627-32.

19. Arora S, Katkov W, Cooley J, et al. Methotrexate in Crohn's disease: results of a randomized, doubleblind, placebo-controlled trial. Hepatogastroenterology 1999;46:1724-9.

20. Oren R, Moshkowitz M, Odes S, et al. Methotrexate in chronic active Crohn's disease: a double-blind, randomized, Israeli multicenter trial. Am J Gastroenterol 1997;92:2203-9.

21. Te HS, Schiano TD, Kuan SF, Hanauer SB, Conjeevaram HS, Baker AL. Hepatic effects of longterm methotrexate use in the treatment of inflammatory bowel disease. Am J Gastroenterol 2000;95: 3150-6.

22. Sandborn WJ, Colombel JF, Enns R, et al. Natalizumab induction and maintenance therapy for Crohn's disease. N Engl J Med 2005;353:1912-25.

23. Targan SR, Feagan BG, Fedorak RN, et al. Natalizumab for the treatment of active Crohn's disease: results of the ENCORE trial. Gastroenterology 2007;132:1672-83.

24. Sands BE, Francis G, Belcher G, et al. The Touch program and risk management plan for the administration of natalizumab: lessons and updated safety results from the use of natalizumab in patients with relapsing multiple sclerosis and implications for potential use in Crohn's disease. Gastroenterology 2008;134:A206.

25. Dassopoulos T, Sninsky CA. Optimizing immuno- 
modulators and anti-TNF agents in the therapy of Crohn disease. Gastroenterol Clin North Am 2012; 41:393-409.

26. Sands BE, Anderson FH, Bernstein CN, et al. Infliximab maintenance therapy for fistulizing Crohn's Disease. N Engl J Med 2004;350:876-85.

27. Colombel JF, Schwartz DA, Sandborn WJ, et al. Adalimumab for the treatment of fistulas in patients with Crohn's Disease. Gut 2009;58:940-8.

28. Ng SC, Plamondon S, Gupta A, et al. Prospective evaluation of anti-TNF therapy guided by MRI for Crohn's perineal fistulas. Am J Gastroenterol 2009; 104:2973-86.

29. Sandborn WJ, Hanauer SB, Rutgeerts P, et al. Adalimumab for maintenance treatment of Crohn's disease: results of the CLASSIC II trial. Gut 2007;56: 1232-9.

30. Hanauer SB, Rutgeerts PJ, D'Haens G, et al. Delayed hypersensitivity to infliximab (Remicade) reinfusion after 2-4 year interval without treatment. Gastroenterology 1999;116:A731.

31. Hanauer SB, Feagan BG, Lichtenstein GR, et al. Maintenance infliximab for Crohn's disease: the ACCENT I randomised trial. Lancet 2002;359: 1541-9.

32. Sandborn WJ, Feagan BG, Stoinov S, et al. Certolizumab pegol for the treatment of Crohn's disease. N Engl J Med 2007;357:228-38.

33. Siegel CA, Marden SM, Persing SM, Larson RJ, Sands BE. Risk of lymphoma associated with combination anti-tumor necrosis factor and immunomodulator therapy for the treatment of Crohn's disease: a meta-analysis. Clin Gastroenterol Hepatol 2009;7:874-81.

34. Deepak P, Sifuentes H, Sherid M, Stobaugh D, Sadozai Y, Ehrenpreis ED. T cell non-Hodgkin's lymphomas reported to the FDA AERS with tumor necrosis factor-alpha (TNF- $\alpha$ ) inhibitors: results of the REFURBISH study. Am J Gastroenterol 2013; 108:99-105.

35. Nozaki K, Silver RM, Stickle DE, et al. Neurological deficits during treatment with tumor necrosis factoralpha antagonists. Am J Med Sci 2011;342:352-5.

36. Derijks LJ, Gilissen LP, Hooymans PM, Hommes DW. Review article: thiopurines in inflammatory bowel disease. Aliment Pharmacol Ther 2006;24: 715-29.

37. Carter MJ, Lobo AJ, Travis SP; IBD Section, British Society of Gastroenterology. Guidelines for the management of inflammatory bowel disease in adults. Gut 2004;53(Suppl 5):V1-16.

38. Wallace TM, Veldhuyzen van Santen SJ. Frequency of use and standards of care for the use of azathioprine and 6-mercaptopurine in the treatment of inflammatory bowel disease: a systematic review of the literature and a survey of Canadian gastroenterologists. Can J Gastroenterol 2001;15:21-8.
39. Azasan package insert. Raleigh, NC: Salix Pharmaceuticals, Inc; 2011.

40. Alstead EM, Ritchie JK, Lennard-Jones JE, Farthing MJ, Clark ML. Safety of azathioprine in pregnancy in inflammatory bowel disease. Gastroenterology 1990;99:443-6.

41. Casanova MJ, Chaparro M, Domenech E, et al. Safety of thiopurines and anti-TNF alpha drugs during pregnancy with inflammatory bowel disease. Am J Gastroenterol 2013;108:433-40.

42. Francella A, Dyan A, Bodian C, Rubin P, Chapman M, Present DH. The safety of 6-mercaptopurine for childbearing patients with inflammatory bowel disease: a retrospective cohort study. Gastroenterology 2003;124:9-17.

43. Fonager K, Sorensen HT, Olsen J, Dahlerup JF, Rasmussen SF. Pregnancy outcome for women with Crohn's disease: a follow-up study based on linkage between national registries. Am J Gastroenterol 1998;93:2426-30.

44. Mahadevan U. Pregnancy and inflammatory bowel disease. Gastroenterol Clin North Am 2009;38: $629-49$.

45. Christensen LA, Dahlerup JF, Nielsen MJ, Fallingborg JF, Schmiegelow K. Azathioprine treatment during lactation. Aliment Pharmacol Ther 2008;28: 1209-13.

46. Moretti ME, Verjee Z, Ito S, Koren G. Breastfeeding during maternal use of azathioprine. Ann Pharmacother 2006;40:2269-72.

47. Gardiner SJ, Gearry RB, Roberts RL, Zhang M, Barclay ML, Begg EJ. Exposure to thiopurine drugs through breast milk is low based on metabolite concentrations in mother-infant pairs. Br J Clin Pharmacol 2006;62:453-6.

48. Sau A, Clarke S, Bass J, Kaiser A, Marinaki A, Nelson-Piercy C. Azathioprine and breastfeeding: is it safe? BJOG 2007;114:498-501.

49. Mahadevan U, Kane S. American Gastroenterological Association institute technical review on the use of gastrointestinal medications in pregnancy. Gastroenterology 2006;131:283-311.

50. Vasiliauskas EA, Church JA, Silverman N, Barry M, Targan SR, Dubinsky MC. Case report: evidence for transplacental transfer of maternally administered infliximab to the newborn. Clin Gastroenterol Hepatol 2006;4:1255-8.

51. Simister NE. Placental transport of immunoglobulin G. Vaccine 2003;21:3365-9.

52. Banaszkiewicz A, Radzikowski A. More on vaccinations in IBD patients. Am J Gastroenterol 2011;106: 545-6.

53. Mahadevan U, Church J, Vasiliauskas E, Sandborn $W$, Dubinsky M. The effect of maternal peripartum infliximab use on neonatal immune response. Gastroenterology 2008;134:A69. 
54. Mahadevan U, Abreu M. Certolizumab use in pregnancy: low levels detected in cord blood. Gastroenterology 2009;136:A960.

55. Yarur A, Kane SV. Update on pregnancy and breastfeeding in the era of biologics. Dig Liver Dis 2013; 45:787-94.

56. Dejaco C, Mittermaier C, Reinisch W, et al. Azathioprine treatment and male fertility in inflammatory bowel disease. Gastroenterology 2001;121: $1048-53$.

57. Teruel C, López-San Román A, Bermejo F, et al. Outcomes of pregnancies fathered by inflammatory bowel disease patients exposed to thiopurines. Am J Gastroenterol 2010;105:2003-8.

58. Mahadevan U, Terdiman JP, Aron J, et al. Infliximab and semen quality in men with inflammatory bowel disease. Inflamm Bowel Dis 2005;11:395-9.

59. El-Beheiry A, El-Mansy E, Kamel N, Salama N. Methotrexate and fertility in men. Arch Androl 1979;3:177-9.
60. Toovey S, Hudson E, Hendry WF, Levi AJ. Sulphasalazine and male infertility: reversibility and possible mechanism. Gut 1981;22:445-51.

61. Sinclair JA, Wasan SK, Farraye FA. Health maintenance in the inflammatory bowel disease patient. Gastroenterol Clin North Am 2012;41:325-37.

62. Agrawal N, Ollington K, Kaneshiro M, Frenck R, Melmed GY. Are immunosuppressive medications associated with decreased responses to routine immunizations? A systematic review. Vaccine 2012;30: 1413-24.

63. Kane S. Abnormal Pap smears in inflammatory bowel disease. Inflamm Bowel Dis 2008;14: 1158-60.

64. Kane S, Khatibi B, Reddy D. Higher incidence of abnormal Pap smears in women with inflammatory bowel disease. Am J Gastroenterol 2008;103: 631-6.

65. Bodger K. Cost effectiveness of treatments for inflammatory bowel disease. Pharmacoeconomics 2011; 29:387-401. 